\title{
Research the Composite Strain Sensors for CNT/Polymer
}

\author{
Rashmi Kulshrestha
}

\begin{abstract}
CNT means carbon nanotube possess piezoelectric due to that behavior its electrical property changes even with the strain applied on the CNT is at the nanoscale. Due to the advantages of composite strain sensor like their multidirectional sensing capability, high strain sensitivity, high dynamic range, flexibility, and low mass density they are turning out to be an ultimate replacement to conventional strain sensors in various applications. CNT film can also be incorporated into the polymer to form a CNT/polymer composite strain sensor. Some of the most famous polymers employed to fabricate CNT/polymer composite strain sensors are thermoplastic polymers polymethyl methacrylate, polystyrene, etc., thermosetting polymers like epoxy resins, polyurethanes, etc., and polydimethylsiloxane (PDMS). The small addition of CNTs to polymer significantly increases their electromechanical properties by enhancing its tensile strength \& young modulus, which in turn improves their sensing performance. CNT/polymer composite strain sensor exhibits better strain transfer, gauge factor, linearity, repeatability, and cost than that of pure CNT strain sensor. There are different factors which are affecting the performance of CNT/polymer composite strain sensor including quality and quantity of CNT filler particles in composite, type of fabrication technique adopted to form a composite, alignment and agglomerates of CNT in composite, cracks or defect in composite and environmental conditions. This paper reviews the performance of various different types of CNT/polymer composite strain sensors.
\end{abstract}

\section{INTRODUCTION}

The Carbon Nanotubes (CNTs) are the stiffest and strongest materials known due to its seamless hexagonal network and unique covalent bonding. These materials have novel properties such as height tensile strength $(50 \mathrm{GPa}$ or above), high electrical conductivity (approx. $1013 \mathrm{a} / \mathrm{cm}^{2}$ ), elasticity (approx. 1 Tpa), high aspect ratio (approx. 1000:1) and high thermal conductivity $(3500 \mathrm{~W} / \mathrm{m} \mathrm{K})[1]$.

Ning Hu studied that numerical simulation was based on a better three-dimensional statistical resistor network model involving the tunneling effect between neighboring nanotubes and fiber reconstruction models. The behavior of a sensor was made under both strained and compressed strains. Both numerical and experimental results show that a higher resistance or higher ratio of tunnel resistance for the total resistance of the sensor leads to a higher sensor sensitivity[2].

Due to unique properties of CNT based strain sensors are pretty beneficial in various applications like structural health monitoring, wearable electronic gadgets, crack or defect detection in infrastructure, luxury automobiles, biological monitoring of human body parts, and also in highly strain sensitive applications [3].

A hexagonal network is formed by using a strong $\mathrm{sp}^{2}$ hybridization in Carbon atoms of CNTs. Due to this bonding

Revised Manuscript Received on August 05, 2019.

Dr. Rashmi Kulshrestha, Department of Chemistry, Sanskriti University, Uttar Pradesh, India.(Email: sanpubip@gmail.com) high tensile strength is present and due to its low mass density, the stiffness of CNTs is very high (230-725 GPa). The mechanical properties of this material are strongly depending on nanotube structures like tensile strength, modulus of elasticity and toughness. As the aspect ratio increases in CNT material the young modulus is also increasing and as the tube diameter increases tensile strength also increases [4]. The electrical conductivity of CNTs governed by the alignment of hexagonal lattice around the tube axis. When the orientation of hexagons are parallel to the axis of the tube it is labeled as an armchair tube, which is metallic in nature and they are a better conductor than metal such as silver and copper. Another two possible orientations of hexagon i.e. zigzag (one with the hexagons oriented in a circle around the axis of the tube) and chiral (one with hexagon not forming a line around the nanotube) show semiconducting behavior [5]. The unique electromechanical property of CNT is that its band structure changes with mechanical deformation, which is a matter of interest for many researchers to use it in strain sensing applications [6].

\section{TYPES OF POLYMER USED}

Some of the important and most popular polymers used to fabricate $\mathrm{CNT} /$ polymer composite strain sensors are:

$>$ Thermosetting polymers (epoxy resins, polyurethanes)

> Thermoplastic polymers (polymethyl methacrylate (PMMA), polystyrene (PS), polypropylene (PP))

$>\quad$ Polydimethylsiloxane (PDMS) [7].

\section{THE STRAIN SENSITIVITY OF THE COMPOSITE SENSOR}

The sensitivity of any strain sensor is calculated by their gauge factor (GF), which is given bythe change in resistance divided by the strain [7][8].

$$
G=\frac{\frac{\Delta R}{R}}{\varepsilon}
$$

Experimentally, it has been observed that the strain sensitivity of CNT/polymer composite strain sensor is better than that of pure CNTs sensors i.e. without polymers. The best gauge factor of composite strain sensor i.e. 22.4 was reported by using epoxy as polymer. Apart from the type of polymers used there are various other parameters, which are affecting the strain sensitivity of composite sensors [9]. They are: 
Quality and quantity of CNTs used,

$>$ Fabrication technique,

$>\quad$ Type of surfactants

$>$ Type of dispersion technique,

$>$ Alignment of CNTs,

$>$ Agglomerates of CNTs,

$>\quad$ Presence or absence of defects and

$>$ Environmental conditions [10].

A minute variations in all these parameters can change the strain sensitivity of the composite strain sensor.

\section{CNT/POLYMER COMPOSITE STRAIN SENSORS \& RESULTS}

The performance analysis and characteristics curve of $\mathrm{CNT}$ /polymer strain sensors using different types of polymers are mentioned below:

\section{Using thermoplastic polymers:}

a) SWCNT/PMMA composite strain sensors: SWCNT/PMMA composite film can be fabricated by dispersing single-walled Bucky paper film in dimethylformamide (DMF) solvent and then bulk mixing the PMMA polymer to it. The strain sensitivity of SWCNT/PMMA strain sensor with different concentration of SWCNT in PMMA [11]is shown in Figure 1.

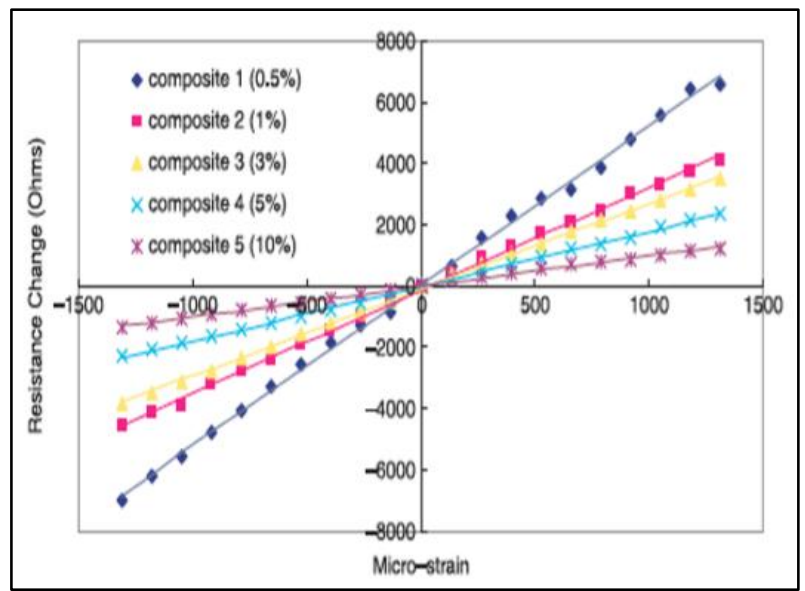

Figure 1. Strain Response of SWCNT/PMMA Composite Strain Sensors[11] to 5 . For $0.5 \%$ wt of SWCNT in PMMA, the gauge factor of composite strain sensor is 5 and it decreases with the increase in CNT content.

b) MWCNT/PMMA composite strain sensor: MWCNT/PMMA composite strain sensor can be fabricated either by dry blended or solution based technique. In dry blended fabrication technique, MWCNTs are dry blended with PMMA powder and then hot pressed multiple times to fabricate the sample. While in the second method, MWCNTs are dissolved in a solvent such as chloroform using mechanical stirrer and then the polymer is added to the solution using ultrasound. Out of these two methods, solution-based technique gives better dispersion and thus produces better strain sensitivity [12] as shown in Figure 2.
Gauge factor of SWCNT/PMMA lies in range between 1

\begin{tabular}{|l|l|l|l|l|l|l|}
\hline $\begin{array}{c}\text { MWCNT } \\
\text { Loading } \\
\text { (wt \%) }\end{array}$ & $\mathbf{1}$ & $\mathbf{3}$ & $\mathbf{5}$ & $\mathbf{6}$ & $\mathbf{8}$ & $\mathbf{1 0}$ \\
\hline $\begin{array}{l}\text { Dry } \\
\text { Blended }\end{array}$ & N/A & N/A & N/A & 8.44 & 7.45 & 5.66 \\
\hline $\begin{array}{l}\text { Solution } \\
\text { Based }\end{array}$ & 15.32 & 4.99 & 4.26 & 3.27 & 1.90 & 1.44 \\
\hline
\end{tabular}

Figure 2. Strain Sensitivity factors of MWNT/PMMA films by using dry blended and solution-based techniques

II. $\quad$ Using thermoplastic polymers [13][14]:

a) MWCNT/epoxy composite strain sensors [13] MWCNT/epoxy composite strain sensors can be manufactured by using two different sorts of MWCNT films i.e. MWCNT-7 and LMWNT-10 film. MWCNT/epoxy composite strain sensors can be fabricated from these two MWCNT films by mixing epoxy and amine hardener by using a planetary mixer and then adding MWCNT films into the mixture and then mixing it again at $2000 \mathrm{rpm}$ for 1 minute. After mixing, the final mixture is then poured into silicon mold to form MWCNT-7/epoxy and LMWNT10/epoxy strain sensors. The piezoresistivity curve of both these sensors [13] are as shown in Figure 3 and

b) Figure 4.

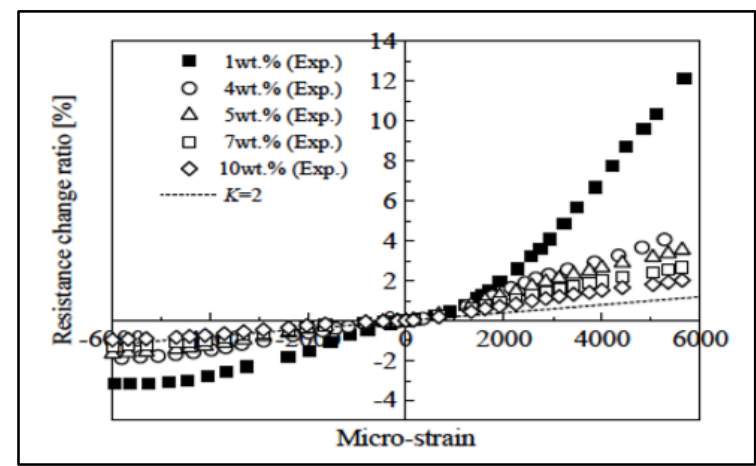

Figure 3. Piezoresistivity of MWCNT-7/epoxy composite strain sensor[13]

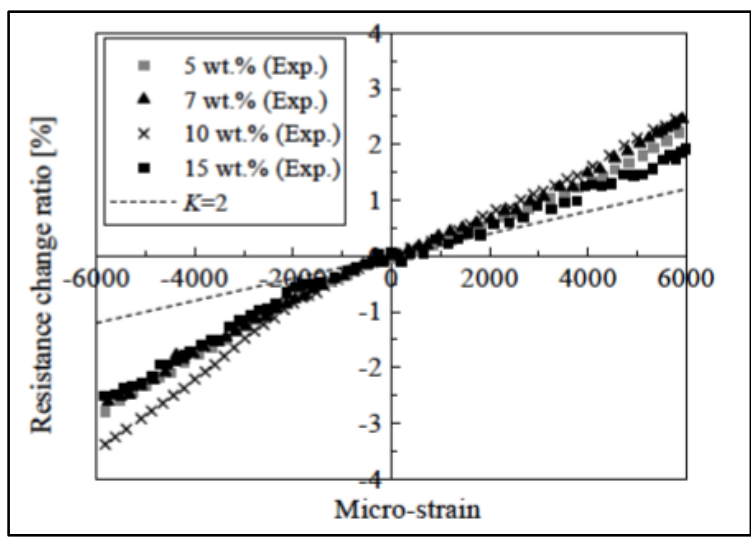

Figure 4. Piezoresistivity of LMWCNT-10/epoxy composite strain sensors[13] 
LMWNT-10 /epoxy composite sensors show linear and symmetric piezoresistivity. On the other hand, MWCNT7/epoxy composite sensor possesses non-linear and antisymmetric piezoresistivity. The gauge factors of MWCNT-7/epoxy and LMWNT10/epoxy strain sensors at a different weight of CNT loading are shown in table 2 and

\begin{tabular}{|c|c|c|c|c|c|c|c|}
\hline \multicolumn{8}{|c|}{$\begin{array}{l}\text { Table 2: Gauge factors of MWNT-7/epoxy strain sensors at } \\
\text { various MWCNT loadings [12]. }\end{array}$} \\
\hline \multicolumn{2}{|c|}{\begin{tabular}{|l|} 
MWCNT Loading (wt \%) \\
\end{tabular}} & \multirow{2}{*}{\multicolumn{2}{|c|}{\begin{tabular}{c|c}
$\mathbf{1}$ \\
7.1
\end{tabular}}} & \multirow{2}{*}{$\begin{array}{c}4 \\
3.5\end{array}$} & \multirow{2}{*}{$\begin{array}{c}5 \\
3.0\end{array}$} & \multirow{2}{*}{$\begin{array}{c}7 \\
2.2\end{array}$} & \multirow{2}{*}{$\begin{array}{c}\mathbf{1 0} \\
2.1\end{array}$} \\
\hline \multirow{2}{*}{\begin{tabular}{|l|} 
Gauge \\
Factors \\
\end{tabular}} & Compressive & & & & & & \\
\hline & Tensile & 7.1 & & 7.6 & 6.2 & 4.8 & $\begin{array}{l}2.1 \\
3.2\end{array}$ \\
\hline \multicolumn{8}{|c|}{$\begin{array}{l}\text { Table 3: Gauge factors of LMWNT-10/epoxy } \\
\text { strain sensors at various MWCNT loadings [12]. }\end{array}$} \\
\hline \multicolumn{3}{|c|}{ MWCNT Loading (wt \%) } & 5 & \multirow{2}{*}{$\begin{array}{c}7 \\
4.1\end{array}$} & 10 & \multicolumn{2}{|r|}{15} \\
\hline \multirow{2}{*}{$\begin{array}{l}\text { Gauge } \\
\text { Factors }\end{array}$} & \multicolumn{2}{|c|}{ Compressive } & 3.8 & & 4.3 & \multicolumn{2}{|r|}{3.2} \\
\hline & \multicolumn{2}{|l|}{ Tensile } & 4.9 & \multicolumn{2}{|c|}{4.5} & \multicolumn{2}{|r|}{4.4} \\
\hline
\end{tabular}

Figure 5. Table 2 and 3

\section{c) MWCNT/PS composite strain sensors [14],}

MWCNT/ PS composite film is fabricated by mixing the functionalized MWCNT with PS solution in toluene. The mixture is stirred to form the homogenous solution, which is then poured onto a flat plate to give flexible MWCNT/PS composite film. The strain sensitivity curve for MWCNT/PS composite film is shown in Figure 6. The gauge factor of MWCNT/PS composite film decreases with the increase in CNT content as shown in Figure 7.

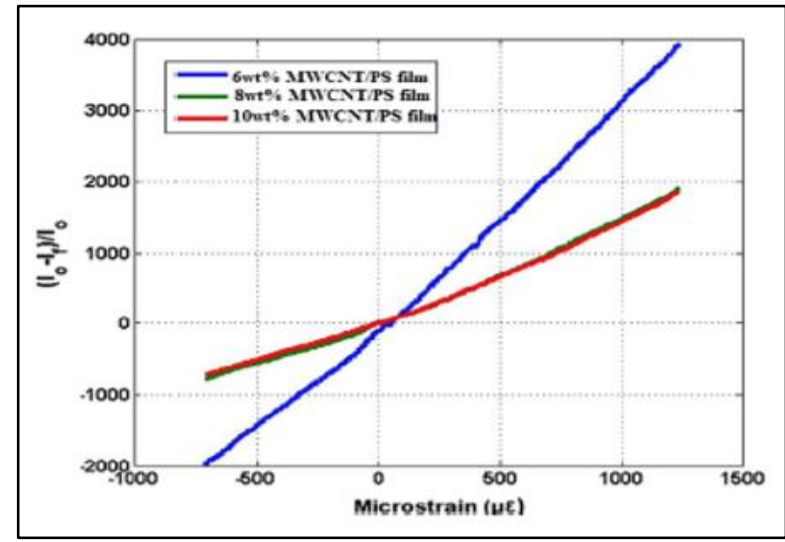

Figure 6. The plot of normalized current response as a function of strain for different wt. \% content of MWCNTs in the MWCNT/PS composite films[14]

\begin{tabular}{|c|c|c|c|c|}
\hline \multicolumn{2}{|c|}{ MWCNT Loading (wt \%) } & 6 & 8 & 10 \\
\hline \multirow{2}{*}{$\begin{array}{l}\text { Gauge } \\
\text { Factors }\end{array}$} & Compressive & 2.55 & 1.04 & 0.998 \\
\hline & Tensile & 3.28 & 1.55 & 1.49 \\
\hline
\end{tabular}

Figure 7. Gauge factors of MWNT/PS films at various MWCNT loadings

III. Using Polydimethylsiloxane (PDMS) polymers[15] [16]:

MWCNT/PDMS composite strain sensors can be manufactured by using two techniques i.e. shear mixing [15] and vacuum filtration [16].In shear mixing technique, MWCNT films are dissolved in the tetrahydrofuran (THF) solvent and sonicated in bath solicitor for 60 minutes. After that PDMS is mixed to the mixture by shear mixing to form the composite film. The production of composite film using table 3 in Figure 5.

vacuum filtration technique comprise the use of MWCNTs film fabricated by chemical vapor deposition (CVD) technique which is then sonicated in dimethylformamide (DMF) solvent and the solution is then ultrasonically treated to disperse the MWCNTs. The final solution is then delivered through polyvinylidene fluoride (PVDF) filter to separate out CNTs in the film. CNTs are then transferred to a flexible substrate by using PDMS molding.

The resistance-strain sensitivity curve of both these samples is shown in Figure 8 and Figure 9. The strain sensitivity is different for both of the sample. MWCNT/PDMS composite sample by shear mixing technique shows a quasi-linear piezoresistive behavior with the gauge factors in the range of 0.8 to 2.3. While the composite fabricated by vacuum filtration shows linear piezoresistivity for a wider range of strain with a lower gauge factor $(0.01$ to 1.25$)$ in comparison to the former sample.

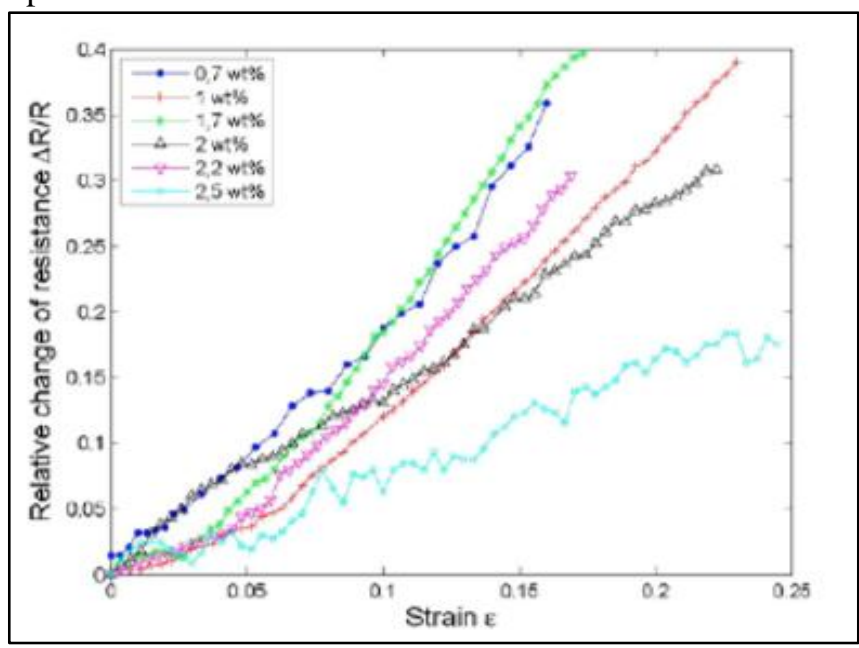

Figure 8. Strain response of MWCNT/PDMS composite strain sensors (shear mixing) for different weight percentages of $\mathrm{MWCN}$ [15]

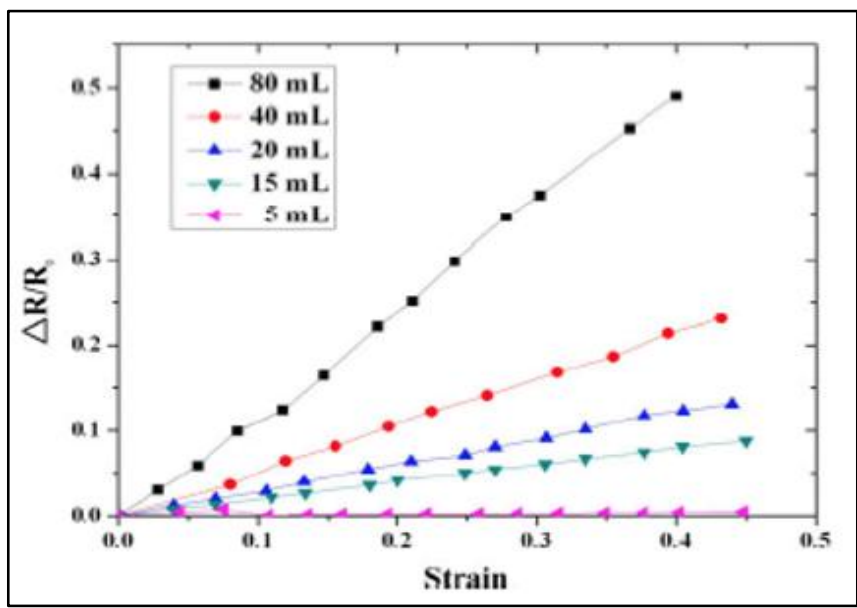

Figure 9. The resistance-strain sensitivity of the CNTs/PDMS thin films (vacuum filtration) with different initial volume of CNTs suspension[16] 


\section{CONCLUSION}

In this paper, $\mathrm{CNT} /$ polymer composite strain sensors have been reviewed. To fabricate $\mathrm{CNT} /$ polymer composite strain sensor, most of the researchers used different type of polymers such as thermoplastic, vinyl, thermosetting, PDMS, etc. in this steady it was discovered that strain sensitivity of CNT/polymer composite strain sensor depends on different parameters such as fabrication parameters, surfactant type, quantity and quality of CNT, polymer type, dispersion method, environmental conditions, defects, alignments and agglomerates of CNTs. By examining the piezoresistive curve of different composite strain sensors it can be determined that they possess highest stain sensitivity in comparison to buck paper, pure CNT and conventional strain sensors. It was also discovered that the sensitivity of the composite strain sensor decreases with the increase in CNT loading. Among all these composite sensors, the best strain sensitivity $(\mathrm{GF} \sim=22.4)$ was achieved with $1 \%$ wt. MWCNT-7/epoxy composite strain sensor with tensile strain. Although there are different researches have been conducted research in the fabrication of CNT/polymer composite strain sensors. But still, there were only very few studies reporting the practical usage of these sensors in commercial applications.

\section{REFERENCES}

1. M. S. Dresselhaus, G. Dresselhaus, and P. Avouris, Carbon nanotubes: synthesis, structure, properties, and applications. Springer, 2001.

2. $\mathrm{N}$. $\mathrm{Hu}$ et al., "Investigation on sensitivity of a polymer/carbon nanotube composite strain sensor," Carbon N. Y., vol. 48, no. 3, pp. 680-687, Mar. 2010.

3. H. Dai, "Carbon Nanotubes: Synthesis, Integration, and Properties," ChemInform, vol. 34, no. 8, Feb. 2003.

4. J. N. Coleman, U. Khan, W. J. Blau, and Y. K. Gun'ko, "Small but strong: A review of the mechanical properties of carbon nanotube-polymer composites," Carbon N. Y., vol. 44, no. 9, pp. 1624-1652, Aug. 2006.

5. A. I. Oliva-Avilés, F. Avilés, and V. Sosa, "Electrical and piezoresistive properties of multi-walled carbon nanotube/polymer composite films aligned by an electric field," Carbon N. Y., vol. 49, no. 9, pp. 2989-2997, Aug. 2011.

6. "(PDF) Electro-mechanical Properties of Carbon Nanotubes." [Online]. Available: https://www.researchgate.net/publication/4679973_Electr o-mechanical_Properties_of_Carbon_Nanotubes.

[Accessed: 04-Jun-2019].

7. M. Kamau and N. Bsc, "CHARACTERISATION OF MULTI WALL CARBON NANOTUBE-POLYMER COMPOSITES FOR STRAIN SENSING APPLICATIONS," 2012.

8. M. Mohiuddin, "Effect of Pressure and Temperature on Electrical Conductivity of CNT-PEEK Composites," 2012.

9. H. Jin and K. Jain, "CARBON NANOTUBE STRAIN SENSORS WITH WIDE DYNAMIC RANGE FABRICATED ON FLEXIBLE SUBSTRATES BY NOVEL PROCESSING TECHNIQUES."

10. K. Parmar, M. Mahmoodi, C. Park, and S. S. Park, "Effect of CNT alignment on the strain sensing capability of carbon nanotube composites," Smart Mater. Struct., vol. 22, no. 7, p. 075006, Jul. 2013.

11. I. Kang et al., "Introduction to carbon nanotube and nanofiber smart materials," Compos. Part B Eng., vol. 37, no. 6, pp. 382-394, Jan. 2006.
12. G. T. ScienceDirect (Online service), Y.-B. Park, Z Liang, C. Zhang, and B. Wang, Composites. Part B, Engineering., vol. 39, no. 1. Elsevier Science Ltd, 1996.

13. G. Gang Yin, N. Ning Hu, Y. Karube, Y. Yaolu Liu, Y. Yuan Li, and H. Fukunaga, "A carbon nanotube/polymer strain sensor with linear and anti-symmetric piezoresistivity," J. Compos. Mater., vol. 45, no. 12, pp. 1315-1323, Jun. 2011

14. R. K. Srivastava et al., "The strain sensing and thermalmechanical behavior of flexible multi-walled carbon nanotube/polystyrene composite films," Carbon N. Y., vol. 49, no. 12, pp. 3928-3936, Oct. 2011.

15. G. Keulemans, F. Ceyssens, M. De Volder, J. W. Seo, and R. Puers, "FABRICATION AND CHARACTERIZATION OF CARBON NANOTUBE COMPO-SITES FOR STRAIN SENSOR APPLICATIONS."

16. X. Song, S. Liu, Z. Gan, Q. Lv, H. Cao, and H. Yan, "Controllable fabrication of carbon nanotube-polymer hybrid thin film for strain sensing," 2009. 